\title{
Environmental enhancement of loose groups around rich clusters of galaxies
}

\author{
M. Einasto ${ }^{1}$, J. Einasto ${ }^{1}$, V. Müller ${ }^{2}$, P. Heinämäki ${ }^{1,3}$, and D. L. Tucker ${ }^{4}$ \\ 1 Tartu Observatory, 61602 Tõravere, Estonia \\ 2 Astrophysical Institute Potsdam, An der Sternwarte 16, 14482 Potsdam, Germany \\ 3 Tuorla Observatory, Väisäläntie 20, Piikkiö, Finland \\ ${ }^{4}$ Fermi National Accelerator Laboratory, MS 127, PO Box 500, Batavia, IL 60510, USA
}

Received 24 January 2002 / Accepted 18 November 2002

\begin{abstract}
We have studied the properties of Las Campanas Loose Groups (Tucker et al. 2000) in the neighbourhood of rich (Abell, APM and X-ray) clusters of galaxies. These loose groups show strong evidence of segregation measured in terms of the group richness and the group velocity dispersion: loose groups in the neighbourhood of a rich cluster are typically 2.5 times more massive and 1.6 times more luminous than groups on average, and these loose groups have velocity dispersions 1.3 times larger than groups on average. This is evidence that the large-scale gravitational field causing the formation of rich clusters enhances the evolution of neighbouring poor systems, a phenomenon recently established in numerical simulations of group and cluster formation.
\end{abstract}

Key words. cosmology: observations - cosmology: large-scale structure of the Universe

\section{Introduction}

On large scales galaxies and clusters form filamentary superclusters, leaving the space between these filamentary structures almost devoid of galaxies. Superclusters and voids form an irregular pattern, which we call the supercluster-void network (Einasto et al. 2001 and references therein). The fine structure of superclusters, as well as the distribution of matter in low density regions between superclusters, can be studied using catalogues of galaxies and of poor systems of galaxies (Lindner et al. 1995). Early wide-field catalogues of galaxies and groups, however, contained only nearby objects (see references in Tucker et al. 2000, hereafter TUC). This has changed recently: several deep surveys of galaxies are now available and can be used for studies of the large scale structure of the Universe in greater detail and on larger scales than ever before. Among these surveys are the ESO Key Program Survey (Vettolani et al. 1997), the Las Campanas Redshift Survey (LCRS; Shectman et al. 1996), the 2 degree Field Galaxy Redshift Survey (2dFGRS) (Colless et al. 2001) and the Sloan Digital Sky Survey (York et al. 2000). On the basis of these and other surveys several deep catalogues of groups have been compiled recently: a catalogue of groups from the ESO Key Program Survey (Ramella et al. 1999), CNOC2 groups

Send offprint requests to: M. Einasto, e-mail: maret@aai.ee
(Carlberg et al. 2001), and groups from the publicly available data of the 2dFGRS survey (Merchan \& Zandivarez 2002).

In this paper, we investigate the environments surrounding the Las Campanas Loose Groups (LCLGs), a catalogue of 1495 loose groups extracted from the LCRS survey (TUC). This catalogue provides an opportunity to study the space distribution and the properties of loose groups on large scales, out to distances of $450 \mathrm{~h}^{-1} \mathrm{Mpc}$.

Rich clusters of galaxies represent high density enhancements in the distribution of galaxies. Therefore, using samples of rich clusters and of neighbouring loose groups, we can study the possible influence of high density environments on the properties of loose groups. In order to pursue this study, we have investigated properties of LCLGs in the vicinity of rich clusters of galaxies, including clusters from the Abell and APM catalogues, X-ray clusters, and even a set of the richest groups from the LCLG catalogue itself. We have then compared the properties of these dense-environment LCLGs with those of typical LCLGs ${ }^{1}$.

In the next section we describe the samples used. In Sect. 3 we extract samples of loose groups near rich clusters, and in Sect. 4 we will study the properties of these loose groups. In the last section we will discuss and summarise of our results.

\footnotetext{
${ }^{1}$ The three-dimensional distribution of LCLGs and rich clusters can be seen by visiting the home page of the Tartu Observatory (http://www.aai.ee/ maret/cosmoweb.html).
} 


\section{Observational data}

\subsection{LCLGs}

The LCRS (Shectman et al. 1996 ) is an optically selected galaxy redshift survey that extends to a redshift of 0.2 and includes 6 slices, each covering an area of roughly $1.5 \times$ 80 degrees. Three of these slices are located in the Northern Galactic Cap and are centred at declinations $\delta=$ $-3^{\circ},-6^{\circ},-12^{\circ}$; the other three slices are located in the Southern Galactic Cap and are centred at declinations $\delta=$ $-39^{\circ},-42^{\circ},-45^{\circ}$. The thickness of the slices is approximately $7.5 h^{-1} \mathrm{Mpc}$ at the survey's median redshift. In all, the LCRS contains 23697 galaxies with redshifts within its official photometric and geometric boundaries.

Survey spectroscopy was first carried out via a 50 fibre multiobject spectrograph, and the nominal apparent magnitude limits for the spectroscopic fields were $16.0 \leq R \leq 17.3$. Partway through the survey, the spectrograph was upgraded to 112 fibres, which permitted a selection of galaxies over the somewhat larger range of apparent magnitudes of $15.0 \leq R \leq$ 17.7. For the sake of efficiency, each LCRS spectroscopic field was observed only once; fields which were observed with the 50-fibre spectrograph were not re-observed. Therefore, the selection criteria varied from field to field, often within a given slice.

Using a friends-of-friends percolation algorithm, TUC extracted the LCLG catalogue from the LCRS. The linking length parameters were chosen so that each group is contained within a galaxy number density enhancement contour of $\delta n / n=80$. In extracting these LCLGs, great care was taken in order to account for both the radial selection function and the field-to-field selection effects inherent in the LCRS. This care is evident in that the derived properties of the LCLGs in the 50-fibre fields do not differ substantially from the derived properties of the LCLGs in the 112-fibre fields (see TUC for details).

The LCLG catalogue contains 1495 groups in a redshift range of $10000 \leq c z \leq 45000 \mathrm{~km} \mathrm{~s}^{-1}$. This is one of the first deep, wide samples of loose groups; as such, it enables us for the first time to investigate the space distribution and properties of groups in a large volume.

\subsection{Abell-class groups from the LCLG catalogue}

We chose the first sample of rich clusters from the LCLG catalogue itself. TUC have calculated an estimate of the Abell counts $N_{\mathrm{ACO}}$ for each group. Among these groups we choose those loose groups with $N_{\mathrm{ACO}} \geq 30$, which corresponds to an Abell richness class of $R=0$.

However, this sample may be affected by selection effects. The study of the mass function of LCLGs (Heinämäki et al. 2003) shows that the sample of loose groups from TUC is complete in the case of groups with masses exceeding $10^{13.5}-10^{14} M_{\odot}$. Thus in order to obtain a complete sample of Abell-class groups from the LCLG list, we excluded from the sample of Abell-class groups all groups with masses less than $10^{13.8} M_{\odot}$, leaving 56 Abell-class LCLGs.

The advantage in using this sample is that these clusters have been determined in the same way as the other groups in the catalogue. Thus, their measured properties can be easily compared with those of other LCLGs without the need to contend with various unknown inter-catalogue systematics. Furthermore, all the neighbours of Abell-class loose groups from the LCLG list are true neighbours, and not mere positional coincidences between rich clusters and loose groups.

\subsection{Abell clusters and superclusters}

We use Abell's catalogue of rich clusters (Abell 1958; Abell et al. 1989), exploiting Andernach \& Tago's (1998) recent compilation of all published redshifts for Abell cluster galaxies. This compilation contains all known Abell clusters with measured redshifts, based on redshifts of individual cluster galaxies, and redshift estimates of clusters according to the formula derived by Peacock \& West (1992), for both Abell catalogues (Abell 1958; Abell et al. 1989). We omitted from the compilation all supplementary, or S-clusters, but included clusters of richness class $R=0$ from the main catalogue. From the general Abell cluster sample we selected all clusters with measured redshifts up to $z_{\mathrm{lim}}=0.13$; beyond this limit the fraction of clusters with measured redshifts becomes small. Our sample contains 1663 clusters, 1071 of which have measured redshifts. We consider that a cluster has a measured redshift if at least one of its member galaxy has a measured redshift. In cases where the cluster has less than three galaxies with measured redshifts, and the measured and estimated redshifts differ by more than a factor of two $\left(\left|\log \left(z_{\text {meas }} / z_{\text {est }}\right)\right|>0.3\right)$, the estimated redshift was used. In this compilation the redshifts of Abell clusters in the region of the LCRS were corrected taking into account the redshift data from the LCRS itself. As a result, in the present study only one Abell cluster has no measured redshift, and this cluster (Abell 2031) is located outside the LCRS borders.

Note that some Abell clusters matched to LCLGs by TUC are absent from our list. TUC used an angular separation criterion to match Abell clusters with LCLGs ( $\Delta \theta<12$ arcmin), whereas physical separation criteria are used here $(\Delta s<$ $6 h^{-1} \mathrm{Mpc}$, as described in the next section). However, the main reason for the absence of some clusters is that loose groups were determined in a narrower redshift range than the whole survey $\left(10000 \leq c z \leq 45000 \mathrm{~km} \mathrm{~s}^{-1}\right.$, while the whole survey extends up to redshifts of about $60000 \mathrm{~km} \mathrm{~s}^{-1}$ ). Nearby and distant clusters that fall within the survey boundaries were of course not included in the present study. Note also that some of the rich clusters that have nearby LCLGs are actually located outside of the borders of LCRS slices. Altogether there are 64 Abell clusters used in the present study, 34 of them are of richness class $R=0$.

The sample of Abell clusters is described in more detail by Einasto et al. (2001), where we present an updated catalogue of superclusters composed of Abell clusters.

\subsection{X-ray selected cluster samples}

We also use X-ray clusters found in the ROSAT All-sky Survey (RASS, Trümper 1993). On the basis of RASS, several catalogues of X-ray selected galaxy clusters have been prepared. 
In the present paper we shall use these samples of X-ray clusters for which the sample volumes are intersected by LCRS slices:

1. Clusters from the all-sky ROSAT Bright Survey of high galactic latitude RASS sources. A detailed description of the data is given in Voges et al. (1999), and the catalogue of X-ray clusters, AGNs, galaxies, small groups of galaxies and other objects is described in Schwope et al. (2000). We shall refer to this sample as the RBS sample;

2. A flux-limited sample of bright clusters from the Southern sky (de Grandi et al. 1999; see also Guzzo et al. 1999 and Borgani \& Guzzo 2001).

All 14 X-ray clusters in the present study have measured redshifts. For details we refer to Einasto et al. (2001).

\subsection{APM clusters}

The APM cluster catalogue (Dalton et al. 1997; hereafter D97) was derived from the APM galaxy catalogue (Maddox et al. 1996), which itself was extracted from plates scanned by the Automatic Plate Measuring (APM) Facility.

The D97 APM cluster catalogue contains 957 clusters, of which 374 have measured redshifts. The redshifts of APM clusters in the region of LCRS were corrected taking into account the redshift data from LCRS. In Einasto et al. (2002) we analysed selection effects in the APM catalogue and found that in the case of APM clusters with estimated redshifts, their space density is artificially enhanced at distances of about 300-350 $h^{-1} \mathrm{Mpc}$ due to selection effects. This property may affect our present analysis. Therefore we decided to use in the present study only those APM clusters with measured velocities.

The APM cluster catalogue contains clusters which are typically poorer than clusters in the Abell catalogue, and have a higher space density. The APM clusters with measured redshifts are less affected by projection effects than Abell clusters (see Einasto et al. 2002). In what follows below, 55 APM clusters provide an additional hunting ground for LCLGs.

For consistency with TUC and our earlier studies of superclusters we calculated distances to groups and clusters using the following formula (Mattig 1958):

$r=\frac{c}{H_{0} q_{0} 2} \frac{q_{0} z+\left(q_{0}-1\right)\left(\sqrt{1+2 q_{0} z}-1\right)}{1+z} ;$

where $c$ is the velocity of light, $H_{0}$ is the Hubble constant, and $q_{0}$ is the deceleration parameter. As in TUC we use $H_{0}=$ $100 h \mathrm{~km} \mathrm{~s}^{-1} \mathrm{Mpc}^{-1}$, and $q_{0}=0.5$. Modern data suggest a model with cosmological constant, which gives about $10 \%$ larger distances than the high-density model used previously.

\section{Populations of LCLGs around rich clusters}

To extract samples of loose groups near rich clusters we searched for LCLGs in spheres around rich clusters using a wide range of neighbourhood radii.

The lower limit of the search radius is determined by the virial radii of the rich clusters and the harmonic radii of the

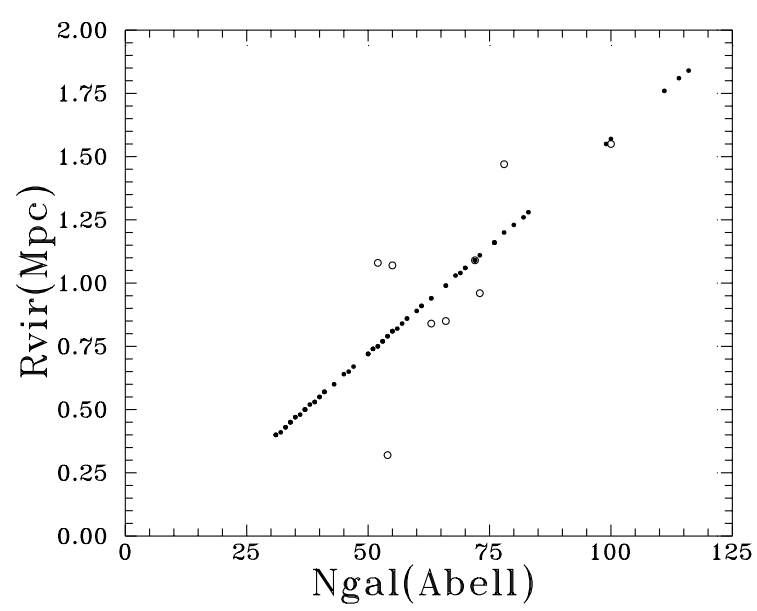

Fig. 1. The estimate of virial radii of Abell clusters (in $h^{-1} \mathrm{Mpc}$ ), against the richness values of these clusters. Open circles: clusters with virial radii from literature (see text). Dots: estimate of virial radii for Abell clusters in our sample.

loose groups. For most rich clusters in our samples virial radii are not available. It is possible to estimate virial radii of clusters using the data about their velocity dispersions (Carlberg et al. 1996) or richness (Mazure et al. 1996). However, the scatter of such estimates is very high; moreover, these estimates are only (relatively) reliable for clusters of richness class $R \geq 1$, whereas about half of the Abell clusters in our samples are of richness class $R=0$.

Thus, we used another approach. We applied virial radii for some Abell clusters in our sample determined by Girardi et al. (1998). These radii are plotted against cluster richness values in Fig. 1. We estimated virial radii for other clusters in our sample using these data and cluster richness values, keeping in mind that we only need estimates of these radii in order to estimate the lower limit of search radius for loose groups near rich clusters. Figure 1 shows that neighbours closer than approximately $1.6 \mathrm{~h}^{-1} \mathrm{Mpc}$ should not be included into our sample. The three richest Abell clusters have larger virial radii estimates, but these clusters do not have very close neighbours among loose groups (the closest neighbour group is located at a distance of $2.7 h^{-1} \mathrm{Mpc}$ from the centre of one of these clusters). We used this limit, $1.6 h^{-1} \mathrm{Mpc}$, in the case of all samples of rich clusters. Additionally, we checked the harmonic radii of loose groups. The loose groups included into our sample all have harmonic radii less than half the distance between a rich cluster and the loose group.

An upper limit of the search radius is determined by the large scale distribution of LCRS galaxies and loose groups in the region of rich clusters. When we increase the search radius, then at certain radii we begin to include into our sample loose groups that are separated from rich clusters by voids. Thus if we simply increase the search radius we obtain populations of groups that actually may not be related to rich cluster. If we want to study the loose groups in the neighbourhood of rich clusters at large radii, we should analyse the region of each cluster individually, and take into account the large scale distribution of galaxies and groups. For the present study we found that an appropriate upper limit for the radius of the neighbour 
search is $6 h^{-1} \mathrm{Mpc}$. By using a search radius of $6 h^{-1} \mathrm{Mpc}$ we obtain a population of loose groups that surround rich clusters and lie within the same high density regions (i.e., within the same superclusters). In some cases, rich clusters within a search radius of $6 h^{-1} \mathrm{Mpc}$ are actually located outside of the borders of LCRS slices.

Additionally, we checked for possible line-of-sight alignments between loose groups and rich clusters using the following test. For a given rich cluster, we searched within a narrow cone for any neighbors within a $\pm 6 h^{-1} \mathrm{Mpc}$ line-of-sight distance from the cluster's location. If there were neighbours in this cone, then in order to be included into the final list of neighbours the distance between the rich cluster and a loose group had to be at least $3.2 h^{-1} \mathrm{Mpc}$ (i.e., twice the original search radius limit described above). With this procedure, the largest number of neighbours excluded from the initial sample - 20 was among the neighbours of APM clusters. According to the loose group richnesses and velocity dispersions, fifteen of these loose groups were possible matches with the APM clusters. In the case of Abell clusters there were six neighbours excluded in this way, which were probable matches between Abell clusters and loose groups (taking into account the parameters of those groups). Around X-ray clusters, as a result of this test we excluded three neighbours from the final sample. And finally, we excluded seven neighbours of Abell-class loose groups located along a line-of-sight with these groups. This procedure excluded all the groups within $2 h^{-1} \mathrm{Mpc}$ of the centre of a rich cluster.

In this way we have defined our samples of loose groups which neighbour rich clusters. To aid our analysis, we have also defined for each of these test samples a corresponding comparison sample consisting of all those loose groups which do not neighbour a rich cluster. We excluded from these comparison samples all loose groups that are within $\pm 6 h^{-1} \mathrm{Mpc}$ of a rich cluster.

We denote samples of LCLGs as follows:

- The sample of groups around Abell-class LCLGs is denoted by LCLG.LG (the corresponding comparison sample by LCLG.cmp.LG);

- The sample of groups around Abell clusters is denoted by LCLG.Abell (the corresponding comparison sample by LCLG.cmp.Abell);

- The sample of groups around APM clusters is denoted by LCLG.APM (the corresponding comparison sample by LCLG.cmp.APM);

- The sample of groups around X-ray clusters is denoted by LCLG.X (the corresponding comparison sample by LCLG.cmp.X).

The lists of LCLGs around Abell, APM and X-ray clusters are given in Tables A.1, 1 and 2.

\section{Properties of LCLGs in the vicinity of rich clusters}

Next, we compare the properties of LCLGs in the cluster environment with the average properties calculated for the comparison samples for each sample under study.
Table 1. The list of LCLG systems around APM clusters.

\begin{tabular}{|c|c|c|c|c|c|}
\hline $\begin{array}{r}N_{\mathrm{APM}} \\
(1) \\
\end{array}$ & $\begin{array}{r}\alpha \\
(2) \\
\end{array}$ & $\begin{array}{r}\delta \\
(3) \\
\end{array}$ & $\begin{array}{r}D \\
(4) \\
\end{array}$ & $\begin{array}{r}N_{\text {LCLG }} \\
(5) \\
\end{array}$ & $\begin{array}{r}\text { Near } \\
(6) \\
\end{array}$ \\
\hline Slice & $=$ & $-39^{\circ}$ & & & \\
\hline 53 & 0020 & -3821 & 328.1 & 8,9 & $\mathrm{~N}$ \\
\hline 160 & 0115 & -3813 & 218.6 & 39 & $\mathrm{~N}$ \\
\hline 162 & 0115 & -3651 & 213.2 & 37 & $\mathrm{~N}$ \\
\hline 172 & 0123 & -3943 & 242.6 & 44 & \\
\hline 173 & 0123 & -3811 & 223.9 & 46,49 & \\
\hline 413 & 0332 & -3915 & 172.4 & $116,117,122$ & $\mathrm{~N}$ \\
\hline 415 & 0332 & -3857 & 177.9 & $111,114,122,123$ & \\
\hline 414 & 0332 & -3934 & 287.0 & 115 & \\
\hline 654 & 2115 & -3947 & 163.9 & 169 & $\mathrm{~N}$ \\
\hline 677 & 2130 & -3847 & 365.4 & 171,174 & $\mathrm{~N}$ \\
\hline 800 & 2234 & -3923 & 207.6 & 198 & $\mathrm{~N}$ \\
\hline 873 & 2313 & -3913 & 186.2 & 223,228 & $\mathrm{~N}$ \\
\hline 914 & 2335 & -3830 & 300.0 & 239 & $\mathrm{~N}$ \\
\hline 944 & 2353 & -3942 & 284.5 & $249,251,256$ & $\mathrm{~N}$ \\
\hline 947 & 2357 & -3946 & 281.9 & 249,256 & $\mathrm{~N}$ \\
\hline Slice & $\delta$ & $-42^{\circ}$ & & & \\
\hline 44 & 0016 & -4203 & 258.4 & 16 & \\
\hline 139 & 0101 & -4307 & 153.1 & $41,42,44$ & \\
\hline 184 & 0129 & -4112 & 245.2 & 51 & $\mathrm{~N}$ \\
\hline 189 & 0130 & -4226 & 242.6 & 50 & $\mathrm{~N}$ \\
\hline 360 & 0314 & -4255 & 183.4 & 92,95 & $\mathrm{~N}$ \\
\hline 365 & 0315 & -4216 & 177.9 & 92,100 & $\mathrm{~N}$ \\
\hline 387 & 0320 & -4130 & 183.4 & 95 & \\
\hline 443 & 0343 & -4121 & 169.6 & $105,106,110$ & \\
\hline 630 & 2103 & -4243 & 300.0 & 140,141 & \\
\hline 644 & 2112 & -4250 & 215.9 & 148,150 & \\
\hline 673 & 2128 & -4330 & 292.3 & 156 & \\
\hline 688 & 2134 & -4119 & 185.9 & 165 & \\
\hline 878 & 2315 & -4238 & 315.4 & 234 & \\
\hline 877 & 2314 & -4257 & 268.9 & 238 & \\
\hline 956 & 2359 & -4407 & 116.7 & 261,5 & $\mathrm{~N}$ \\
\hline Slice & $\delta=$ & $-45^{\circ}$ & & & \\
\hline 19 & 0011 & -4521 & 375.6 & 10 & $\mathrm{~N}$ \\
\hline 139 & 0102 & -4308 & 153.1 & 32 & $\mathrm{~N}$ \\
\hline 185 & 0128 & -4432 & 345.7 & 57 & \\
\hline 238 & 0206 & -4501 & 289.6 & $75,78,81$ & \\
\hline 289 & 0243 & -4526 & 271.5 & 95,101 & \\
\hline 351 & 0312 & -4447 & 315.3 & 111 & $\mathrm{~N}$ \\
\hline 362 & 0314 & -4540 & 202.4 & 112 & $\mathrm{~N}$ \\
\hline 366 & 0315 & -4514 & 213.2 & 114 & \\
\hline 369 & 0316 & -4450 & 215.9 & 114 & $\mathrm{~N}$ \\
\hline 374 & 0316 & -4425 & 205.1 & 112,113 & $\mathrm{~N}$ \\
\hline 433 & 0339 & -4551 & 188.8 & $115,119,120,124,125$ & \\
\hline 450 & 0346 & -4540 & 197.0 & $124,129,130$ & \\
\hline 509 & 0430 & -4613 & 191.5 & 151 & $\mathrm{~N}$ \\
\hline 642 & 2110 & -4446 & 284.5 & 165 & \\
\hline 650 & 2114 & -4509 & 261.1 & 167 & $\mathrm{~N}$ \\
\hline 651 & 2114 & -4541 & 188.9 & 159,173 & \\
\hline 653 & 2115 & -4528 & 271.5 & 169 & \\
\hline 657 & 2116 & -4532 & 276.8 & 169 & \\
\hline 659 & 2118 & -4543 & 271.5 & 169 & \\
\hline 709 & 2144 & -4407 & 178.0 & 184 & $\mathrm{~N}$ \\
\hline 757 & 2209 & -4544 & 328.1 & 198 & $\mathrm{~N}$ \\
\hline 812 & 2241 & -4535 & 253.2 & $217,219,221,223$ & $\mathrm{~N}$ \\
\hline 814 & 2242 & -4521 & 253.2 & $217,219,221,223$ & \\
\hline 825 & 2247 & -4535 & 147.6 & 228,229 & $\mathrm{~N}$ \\
\hline 844 & 2258 & -4416 & 242.6 & 230,235 & \\
\hline 915 & 2337 & -4615 & 191.6 & 250,254 & $\mathrm{~N}$ \\
\hline 956 & 2359 & -4407 & 116.7 & 263 & \\
\hline
\end{tabular}

Columns are given in Table A.1. 
Table 2. The list of LCLG systems around X-ray clusters.

\begin{tabular}{|c|c|c|c|c|c|}
\hline $\begin{array}{r}N_{\mathrm{RBS}} \\
\text { (1) }\end{array}$ & $\begin{array}{r}\alpha \\
(2)\end{array}$ & $\begin{array}{r}\delta \\
(3)\end{array}$ & $\begin{array}{r}D \\
(4)\end{array}$ & $\begin{array}{r}N_{\text {LCLG }} \\
\text { (5) }\end{array}$ & $\begin{array}{r}N_{\text {Abell }} \\
\text { (6) }\end{array}$ \\
\hline Slice & $\delta=$ & $-3^{\circ}$ & & & \\
\hline 1193 & 1256 & -129 & 238.3 & 174 & A 1650 \\
\hline 1197 & 1256 & -355 & 236.7 & 172 & A1651 \\
\hline 1205 & 1300 & -215 & 231.9 & $172,174,176$ & A1663 \\
\hline Slice & $=$ & $-12^{\circ}$ & & & \\
\hline 851 & 1015 & -1027 & 168.6 & 7,8 & A 970 \\
\hline 1020 & 1138 & -1205 & 323.5 & 94 & A1348 \\
\hline 1151 & 1242 & -1143 & 264.9 & $143,144,147$ & A1606 \\
\hline 1337 & 1359 & -1055 & 198.1 & 218 & A1837 \\
\hline Slice & $=$ & $-39^{\circ}$ & & & \\
\hline 521 & 0414 & -3806 & 143.9 & $141,142,143,147$ & $\mathrm{nA}$ \\
\hline Slice & $=$ & $-42^{\circ}$ & & & \\
\hline 469 & 0345 & -4112 & 172.2 & $105,106,110$ & A0384 \\
\hline 1990 & 2318 & -4210 & 252.1 & 236,237 & A3998 \\
\hline Slice & $=$ & $-45^{\circ}$ & & & \\
\hline 459 & 0340 & -4541 & 191.3 & $115,120,127$ & $\mathrm{nA}$ \\
\hline 1782 & 2143 & -4408 & 177.7 & 187 & A3809 \\
\hline $\mathrm{Rx} 31$ & 0312 & -4536 & 206.7 & 112 & A3104 \\
\hline
\end{tabular}

Columns are given in Table A.1.

In the LCLG catalogue several physical properties have been calculated for each group (TUC, Sect. 4). These include the observed number of group member galaxies $N_{\text {obs }}$, the harmonic radius $R_{\mathrm{h}}$, the line-of-sight velocity dispersion $\sigma_{\text {los }}$, the virial mass $M_{\mathrm{vir}}$, the total luminosity $L_{\mathrm{tot}}$, and the Abell counts $N_{\mathrm{ACO}}$.

Each of these properties highlights a different physical aspect of groups. Thus, it is useful to analyse how several of these properties differ between a sample of groups in high-density environments and for the comparison sample. We refer to TUC for details of how these properties were estimated for the LCLG catalogue. Now let us analyse the properties of loose groups in more detail.

The results of our calculations are shown in Table 3, where we give the median and upper quartile values of the properties of groups from various samples. The cumulative distributions of the properties of loose groups are plotted in Figs. 2-7. We have also estimated the statistical significance of our results with the Kolmogorov-Smirnov test (Table 4).

First, consider the distribution of observed group richness values (number of galaxies) shown in Fig. 2. In most cases, an LCLG near a rich cluster typically has a larger observed group membership than loose groups from the comparison catalogue. Note that the differences between the test and the comparison samples are larger for the upper quartiles (Table 3).

The observed membership of loose groups, however, is fraught with selection effects. A better measure of a group's richness is its Abell count $N_{\mathrm{ACO}}$. To first order, the measured Abell counts for LCLGs should be independent of selection effects (see TUC). When using the Abell counts as the measure of group richness, a LCLG near a rich cluster tends to be richer than a typical loose group drawn from the comparison sample (Fig. 3).

Figure 4 shows that the harmonic radii of loose groups in the neighbourhood of rich clusters are somewhat larger than those of loose groups on average in the comparison sample. The largest differences occur between loose groups around Abell-class LCLGs and the smallest differences in the case of loose groups around Abell and APM clusters.

Figure 5 shows that the velocity dispersion of loose groups in the neighbourhood of rich clusters is about 1.3 times larger than that in the comparison samples (these groups are "hotter"). The upper quartile values for velocity dispersions of groups in the neighbourhood of X-ray clusters are especially large.

Figure 6 shows the distribution of masses of loose groups in the vicinity of rich clusters. This figure shows the presence of a strong mass segregation - loose groups in the vicinity of rich clusters have masses that are, on average, about 2.5 times larger than mean masses of loose groups.

Figure 7 shows that loose groups in the neighbourhood of Abell-class loose groups and in the neighbourhood of Abell clusters are 1.6 times more luminous than loose groups from comparison samples. In the case of loose groups around $\mathrm{X}$-ray clusters the upper quartile values show a large difference between dense-environment groups and the comparison sample. The smallest differences are found for loose groups around APM clusters.

Next let us check whether the properties of loose groups around rich clusters depend on the properties of the rich clusters themselves.

First, we consider the richness of Abell and APM clusters. In Fig. 8 we plot the masses of loose groups around Abell clusters against the Abell cluster richnesses (the results for APM clusters are similar, but to avoid overcrowding they are not shown). Figure 8 shows no clear dependence on the cluster's richness. We obtain a similar result if we plot group properties other than mass.

In Fig. 9 we show the masses of loose groups around Abellclass loose groups against the masses of these Abell-class loose groups themselves. Again, this figure shows no clear dependence of a loose group's mass on the mass of the nearby Abellclass group.

Summarising, these figures demonstrate a strong environmental enhancement of the mass and the richness of loose groups in the vicinity of rich clusters of galaxies. The differences between properties of loose groups in the neighbourhood of rich clusters and those of loose groups away from rich clusters are minimal in the case of loose groups around APM clusters. This may be due to the fact that APM clusters are themselves poorer than rich clusters from the other samples. Somewhat lower confidence levels in the statistical significance of the differences between samples in the case of loose groups around X-ray clusters (Table 4) are likely due to the small number of loose groups in this sample. Finally, we note that, although loose group properties do appear to depend on proximity to rich clusters, they do not appear to depend on the richness of rich clusters themselves. 
Table 3. Median and upper quartile (in parentheses) values of LCLG properties.

\begin{tabular}{|c|c|c|c|c|c|c|c|}
\hline Sample & $N_{\text {group }}$ & $N_{\text {obs }}$ & $N_{\mathrm{ACO}}$ & $\begin{array}{r}R_{\mathrm{h}} \\
h^{-1} \mathrm{Mpc} \\
(5)\end{array}$ & $\begin{array}{r}\sigma_{\text {los }} \\
\mathrm{km} \mathrm{s}^{-1} \\
(6)\end{array}$ & $\begin{array}{r}\log M_{\text {vir }} \\
h^{-1} M_{\odot} \\
\text { (7) }\end{array}$ & $\begin{array}{r}\log L_{\text {tot }} \\
h^{-2} L_{\odot} \\
(8)\end{array}$ \\
\hline LCLG.LG & 95 & $4.5(7.0)$ & $19.5(28.0)$ & $0.65(0.89)$ & $235(295)$ & $13.65(14.00)$ & $11.35(11.65)$ \\
\hline LCLG.cmp.LG & 1340 & $4.0(5.5)$ & $15.5(25.5)$ & $0.46(0.75)$ & $175(275)$ & $13.20(13.75)$ & $11.15(11.35)$ \\
\hline LCLG.Abell & 96 & $5.5(9.5)$ & $18.5(30.0)$ & $0.51(0.78)$ & $215(300)$ & 13.55 (13.90) & $11.25(11.60)$ \\
\hline LCLG.cmp.Abell & 1379 & $4.5(5.5)$ & $16.5(24.5)$ & $0.46(0.76)$ & $180(265)$ & $13.25(13.75)$ & $11.15(11.45)$ \\
\hline LCLG.X & 24 & $6.0(10.0)$ & $15.0(30.0)$ & $0.54(0.80)$ & $225(400)$ & $13.65(14.15)$ & $11.15(11.75)$ \\
\hline LCLG.cmp.X & 1465 & $4.5(5.5)$ & $15.5(25.5)$ & $0.46(0.77)$ & $180(265)$ & $13.25(13.75)$ & $11.15(11.45)$ \\
\hline LCLG.APM & 85 & $4.5(7.5)$ & $14.5(26.0)$ & $0.55(0.81)$ & $210(295)$ & $13.55(13.90)$ & $11.15(11.55)$ \\
\hline LCLG.cmp.APM & 1377 & $4.5(5.5)$ & $16.5(24.5)$ & $0.46(0.77)$ & $180(265)$ & $13.25(13.75)$ & $11.15(11.45)$ \\
\hline
\end{tabular}

Endnotes: the columns are as follows:

Column 1: sample identification, given in Sect. 3.

Column 2: $N_{\text {group }}$, the number of LCLGs in the sample.

Column 3: $N_{\mathrm{obs}}$, median value of the observed number of LCRS galaxies in groups.

Column 4: $N_{\mathrm{ACO}}$, median value of the group's Abell counts.

Column 5: $R_{\mathrm{h}}$, median value of the harmonic radius of the groups, in units of $h^{-1} \mathrm{Mpc}$.

Column 6: $\sigma_{\text {los }}$, median value of the group line-of-sight velocity dispersions, in units of $\mathrm{km} \mathrm{s}^{-1}$.

Column 7: $M_{\text {vir }}$, median value of the group's virial mass, in units of $h^{-1} M_{\odot}$.

Column 8: $L_{\text {tot }}$, the median value of the total group luminosity in the LCRS $R$-band, in units of solar luminosity $\left(h^{-2} L_{\odot}\right)$.
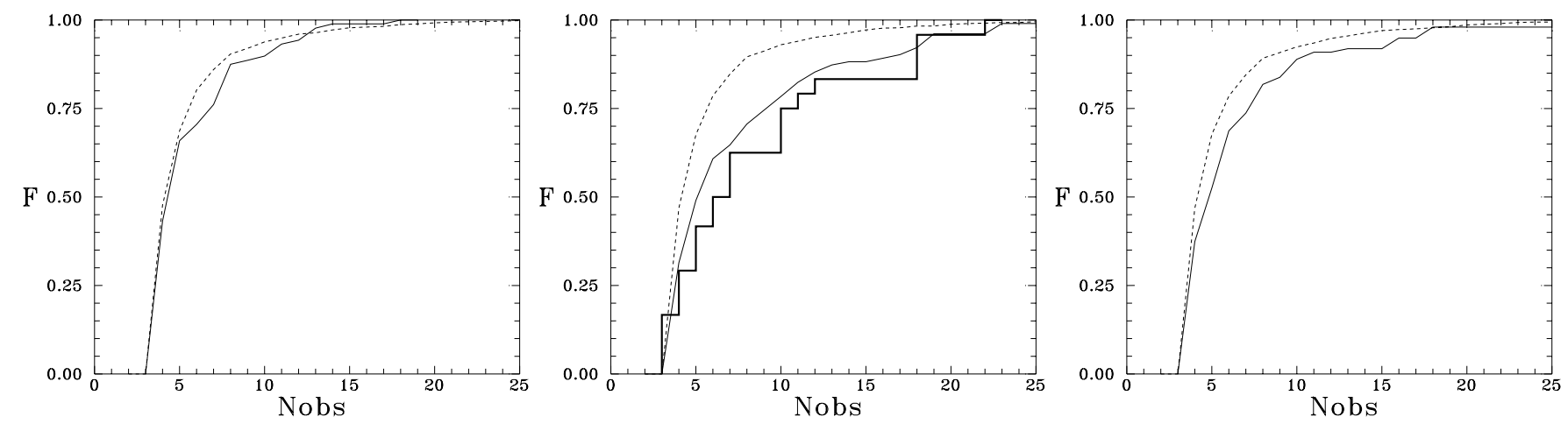

Fig. 2. The distribution of the observed group membership (observed numbers of galaxies) for LCLGs near a rich cluster. In all three panels, the dashed line represents the distribution for groups from comparison sample (see text; in the middle panel the comparison sample for loose groups around X-ray clusters is not plotted since it almost coincides with the comparison sample for Abell clusters). Left panel: solid line: LCLGs around Abell-class LCLGs $\left(N_{\mathrm{ACO}} \geq 30\right.$ ). Middle panel: LCLGs around Abell clusters (thin solid line) and X-ray clusters (bold solid line). Right panel: LCLGs around APM clusters (the solid line).
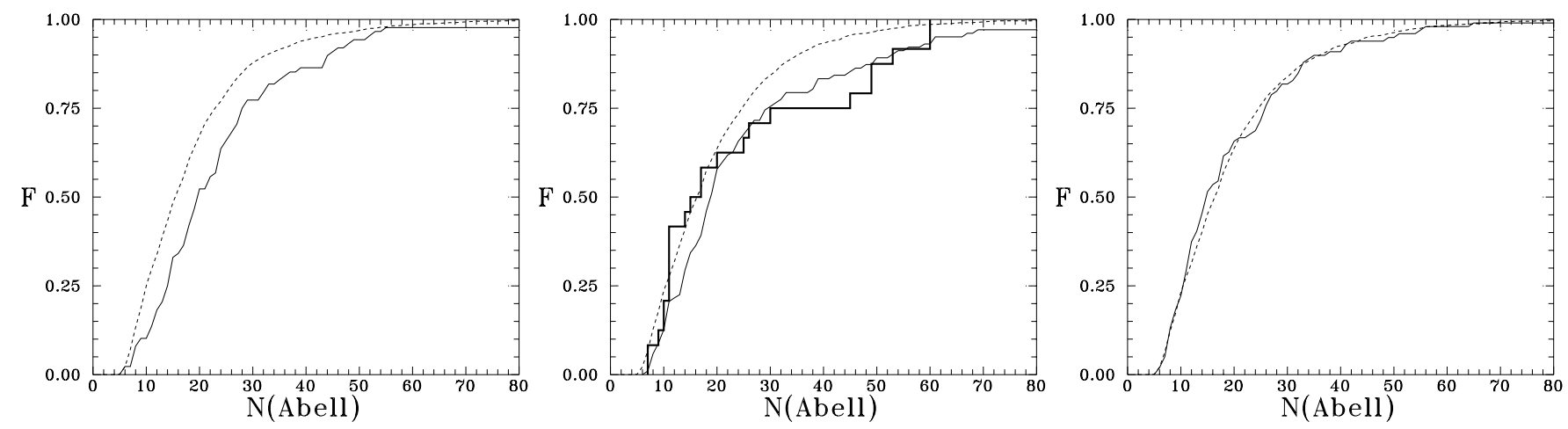

Fig. 3. Distribution of Abell counts for LCLGs around rich clusters. Panels and lines are as in Fig. 2. 

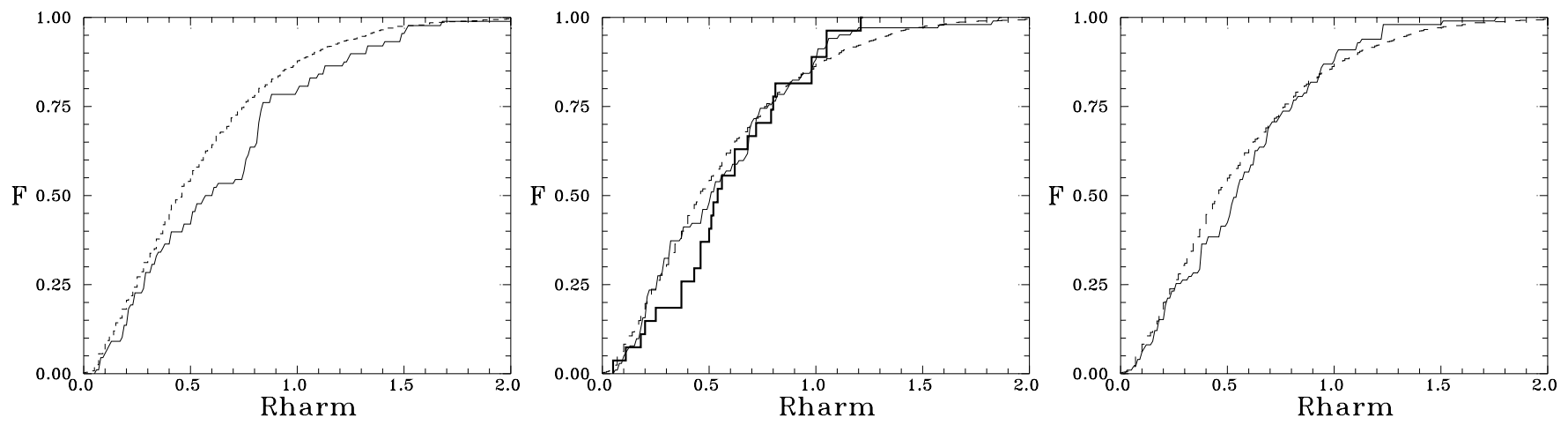

Fig. 4. Distribution of harmonic radii (in $h^{-1} \mathrm{Mpc}$ ) for LCLGs around rich clusters. Panels and lines are as in Fig. 2.
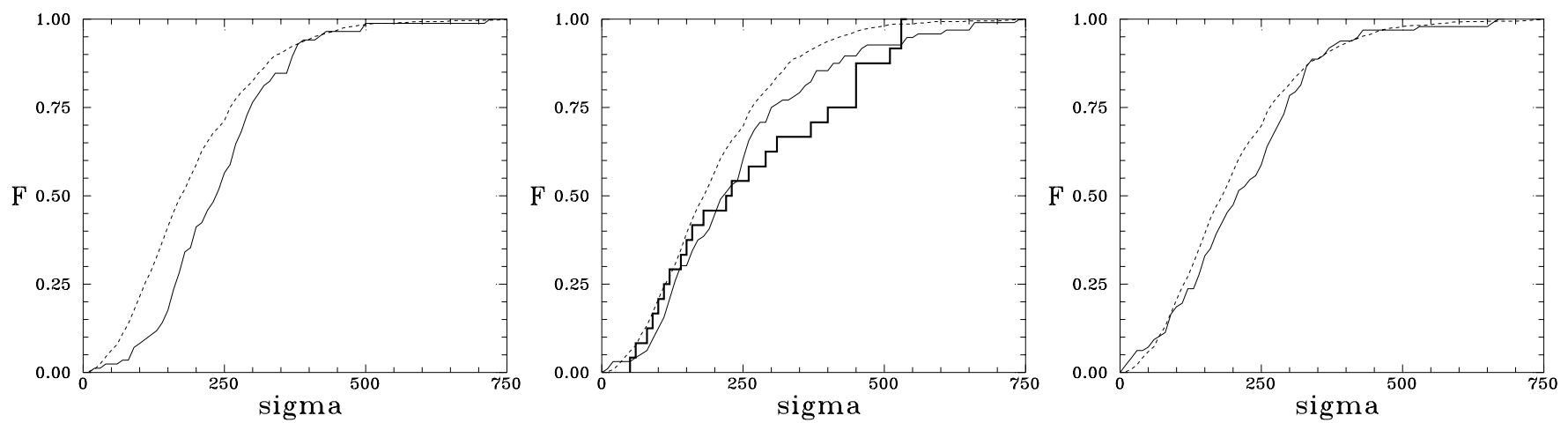

Fig. 5. Distribution of velocity dispersions, $\sigma_{\text {los }}\left(\mathrm{km} \mathrm{s}^{-1}\right)$ for LCLGs around rich clusters. Panels and lines are as in Fig. 2.
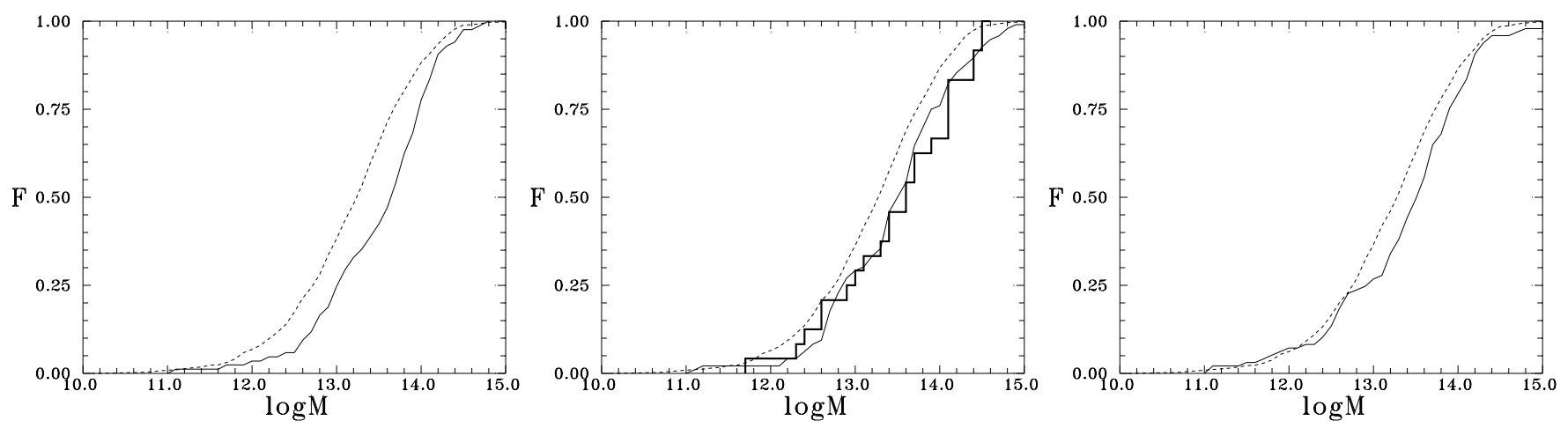

Fig. 6. Distribution of masses $\left(h^{-1} M_{\odot}\right)$ for LCLGs around rich clusters. Panels and lines are as in Fig. 2.

\section{Discussion and conclusions}

We have analysed the properties of loose groups from the LCRS in high density regions as determined by the location of rich optical and X-ray clusters. Our study demonstrates an environmental enhancement of some characteristic properties of these groups: they are richer, more massive, and more luminous than typical loose groups on average. Now we shall discuss the confidence of our results. First we consider selection effects.

\subsection{The properties of loose groups and selection effects}

There are two main selection effects in the LCRS: in apparent magnitude, and an almost random selection due to the limited number of fibres used in the survey (see also TUC; Doroshkevich et al. 2001; Heinämäki et al. 2003).

LCRS observations were performed in a fixed apparent magnitude interval and therefore the absolute magnitude interval of observed galaxies depends on the distance of the galaxy - galaxies fainter or brighter than this interval are not included in the survey. Thus groups consisting of faint galaxies occur only in the nearest regions of the survey. With increasing distance, groups containing fainter galaxies gradually disappear from the sample. This effect is seen in Fig. 10 where we plot the total luminosity of loose groups against distances of these groups for two samples: the total sample of LCLGs and the sample containing only those loose groups around Abell-class groups (for other samples of loose groups in the environment of rich clusters the distribution is similar). 

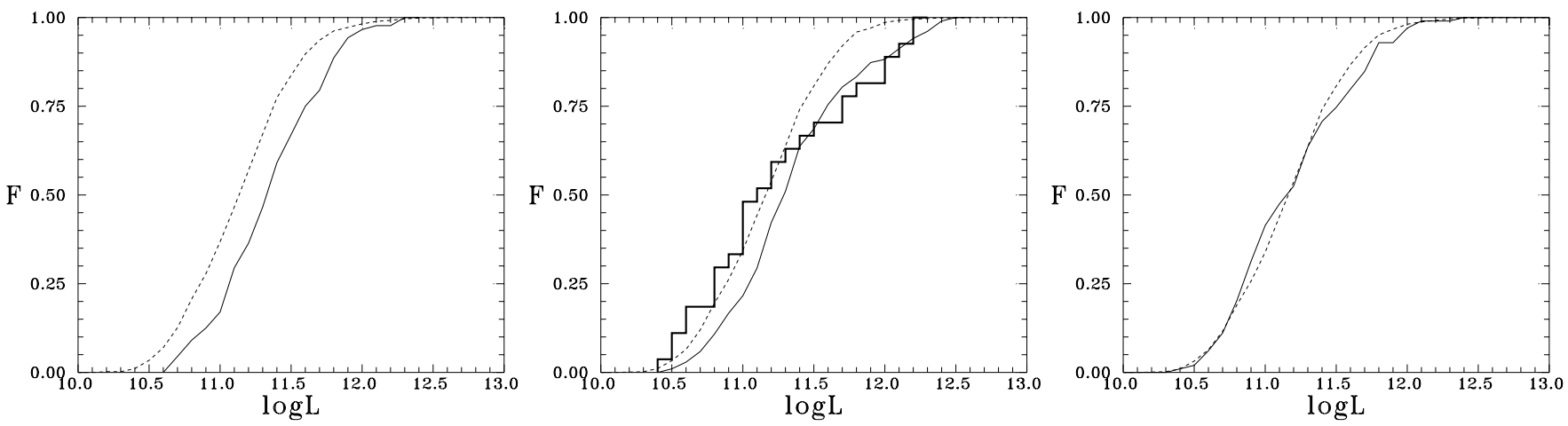

Fig. 7. Distribution of luminosities (in units of solar luminosity $h^{-2} L_{\odot}$ ) for LCLGs around rich clusters. Panels and lines are as in Fig. 2.

Table 4. Confidence levels of the statistical significance of the Kolmogorov-Smirnov tests.

\begin{tabular}{lrrrrrrr}
\hline \hline Sample & $N_{\text {group }}$ & $N_{\text {obs }}$ & $N_{\text {ACO }}$ & $R_{\mathrm{h}}$ & $\sigma_{\text {los }}$ & $\log M_{\text {vir }}$ & $\log L_{\text {tot }}$ \\
$(1)$ & $(2)$ & $(3)$ & $(4)$ & $(5)$ & $(6)$ & $(7)$ & $(8)$ \\
\hline LCLG.LG & 95 & $75 \%$ & $99 \%$ & $99 \%$ & $98 \%$ & $99 \%$ & $99 \%$ \\
LCLG.Abell & 96 & $99 \%$ & $90 \%$ & $75 \%$ & $75 \%$ & $97 \%$ & $95 \%$ \\
LCLG.X & 24 & $99 \%$ & $75 \%$ & $70 \%$ & $85 \%$ & $80 \%$ & $70 \%$ \\
LCLG.APM & 85 & $90 \%$ & $65 \%$ & $75 \%$ & $90 \%$ & $90 \%$ & $65 \%$ \\
\hline
\end{tabular}

Endnotes: the columns are as follows:

Column 1: sample identification, given in Sect. 3.

Column 2: $N_{\text {group }}$, the number of LCLGs in the sample.

Column 3: KS statistical significance for $N_{\text {obs }}$.

Column 4: KS statistical significance for $N_{\mathrm{ACO}}$.

Column 5: KS statistical significance for $R_{\mathrm{h}}$.

Column 6: KS statistical significance for $\sigma_{\text {los }}$.

Column 7: KS statistical significance for $M_{\text {vir }}$.

Column 8: KS statistical significance for $L_{\text {tot }}$.

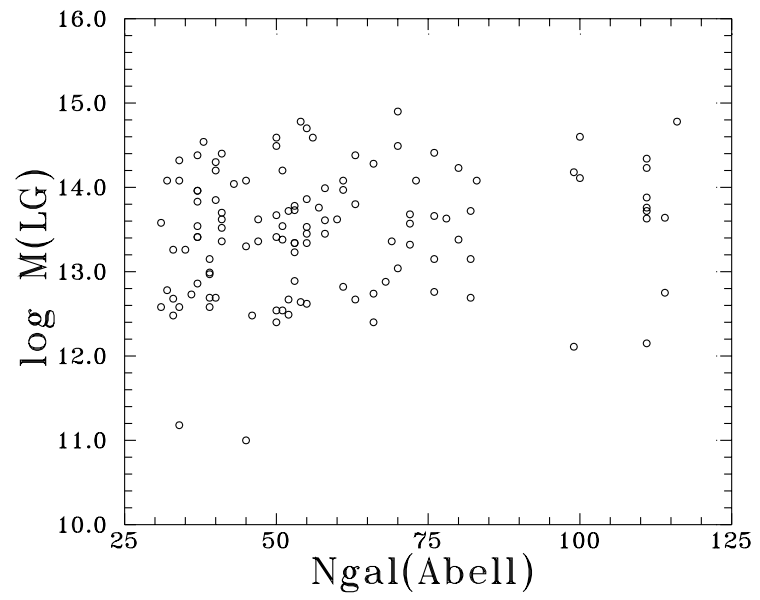

Fig. 8. Masses of LCLGs around Abell clusters (sample LCLG.Abell, masses are in units of $h^{-1} M_{\odot}$ ), against the richnesses of these Abell clusters.

This figure shows that this effect is similar both for loose groups around rich clusters and for the total sample of loose groups. However, galaxies in low density environments tend to be fainter (Hamilton 1988; Einasto 1991; Lindner et al. 1995; Norberg et al. 2001; Zehavi et al. 2002), and thus at a given distance the probability that a galaxy may not be observed in the

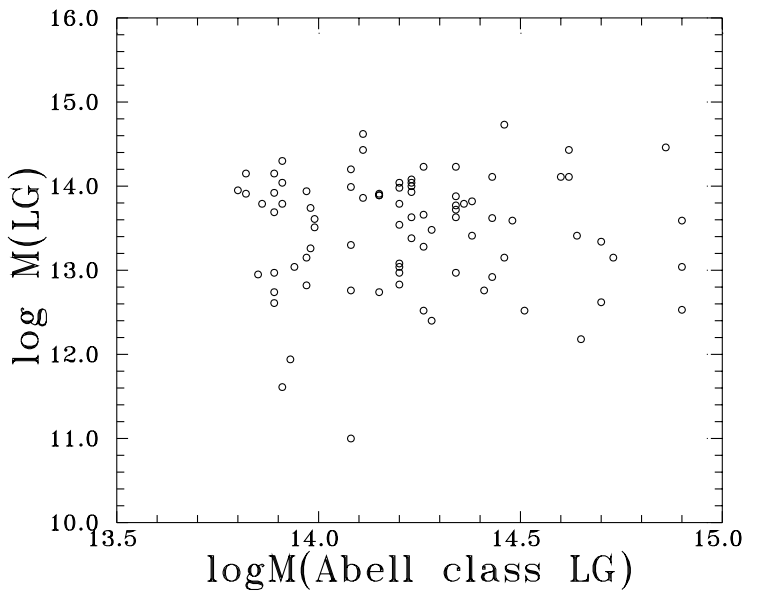

Fig. 9. Masses of LCLGs around Abell-class loose groups (sample LCLG.LG), against the masses of these Abell-class groups (in units of $h^{-1} M_{\odot}$ ).

LCRS window of apparent magnitudes is higher in low density regions. Thus loose groups in the high density environments studied in the present paper are probably less affected by the luminosity selection effect than loose groups on average.

However, Fig. 10 shows that there are no loose groups in the neighbourhood of rich clusters among the nearest groups 
included in the comparison sample. Could it be possible that the environmental effect we study in the present paper is simply a selection effect due to the luminosity-distance relation?

To clarify this problem we choose both samples of loose groups around rich clusters and comparison samples from three distance intervals: $150-400 h^{-1} \mathrm{Mpc}, 200-400 h^{-1} \mathrm{Mpc}$, and 250-400 $h^{-1} \mathrm{Mpc}$. Then we derived the distribution of the properties of these distance-limited samples. In Table 5 we give the median and upper quartile values of the properties of groups from these distance intervals in the case of loose groups around Abell-class groups (sample LCLG.LG). Figure 11 shows the cumulative distribution of masses of groups for samples of loose groups around Abell-class loose groups, and for corresponding comparison samples from different distance intervals. In the case of loose groups around other rich clusters the results are similar.

Table 5 and Fig. 11 show clearly that the selection effect due to the luminosity-distance relation does not change our main conclusion: properties of groups in the neighbourhood of rich clusters are enhanced in comparison with the properties of loose groups on average.

The Kolmogorov-Smirnov test shows that the differences between the samples are statistically significant. For example, the distributions of masses for samples from distance interval 250-400 $h^{-1} \mathrm{Mpc}$ are different at the $95 \%$ confidence level.

Another selection effect in the LCRS is due to different number of fibres used during redshift measurements: depending on the field, 50 or 112 galaxies were measured for redshifts, but the actual number of galaxies in the magnitude window is larger, and the sample is diluted. Dilution decreases not only the number of galaxies in systems but also the number of groups as the number of galaxies in the group may fall below the critical value of 3 used in the group search (see Heinämäki et al. 2003). In high-density environments this effect may be stronger than in low-density environments. When compiling the list of loose groups, TUC attempted to take this effect into account. However there is no simple and obvious way to correct for this selection effect for very poor groups.

Recently, Doroshkevich et al. (2001) analysed selection effects in the LCRS catalogue and concluded that selection effects are stronger in low density environments; in high density environments these effects may serve merely to decrease the observed richnesses of galaxy systems.

\subsection{The local number density test for loose groups}

So far the populations of loose groups were selected in the high density environments determined by the neighbourhood of rich clusters of galaxies. Now we check the possibility that loose groups in all high density regions (and not just in the neighbourhood of rich clusters) are richer, have higher velocity dispersions and so on. For that we select loose groups from high density environments as determined by the number density of loose groups themselves.

The local density around loose groups can be determined by the number density of neighbours around loose groups in a spheres of a given radius. We searched for close neighbours for

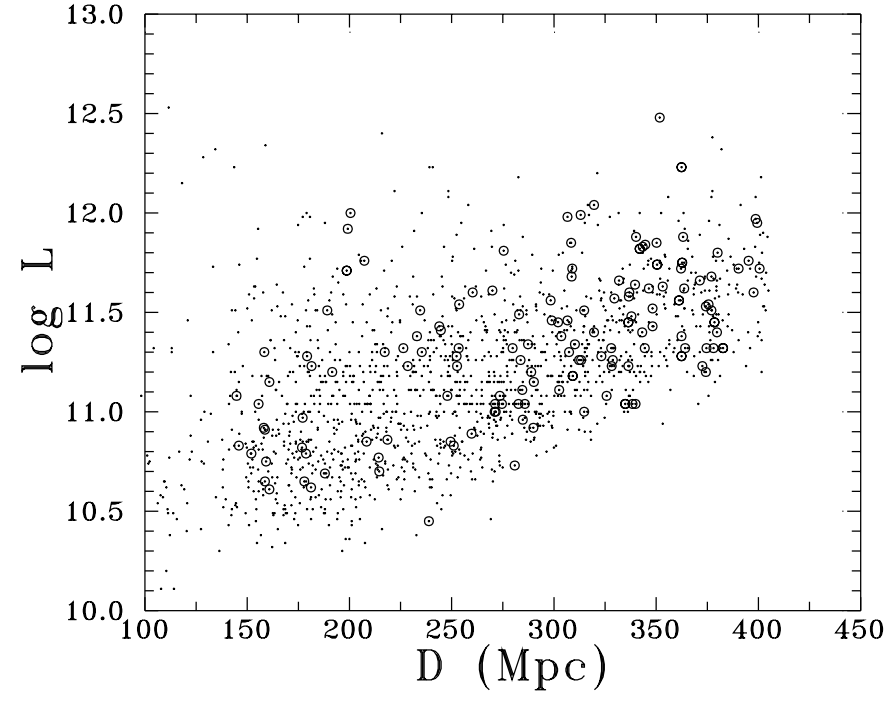

Fig. 10. Luminosities of LCRS loose groups from total sample of the LCLGs (dots) and from the sample of groups near Abell-class loose groups (open circles) (in units of solar luminosity $h^{-2} L_{\odot}$ ) against group's distances (in $h^{-1} \mathrm{Mpc}$ ).

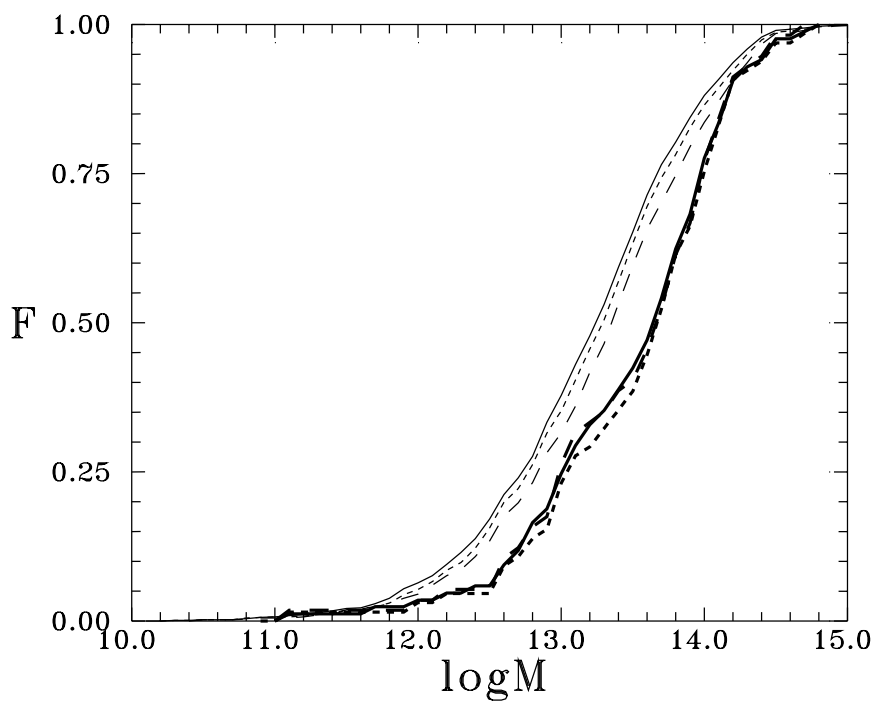

Fig. 11. Distribution of masses $\left(h^{-1} M_{\odot}\right)$ for LCLGs around Abellclass loose groups (bold lines), and for loose groups from comparison samples (thin lines). Solid lines: samples from distance interval 150 $400 h^{-1} \mathrm{Mpc}$, short-dashed lines: samples from distance interval 200$400 \mathrm{~h}^{-1} \mathrm{Mpc}$, and long-dashed lines: samples from distance interval 250-400 $h^{-1}$ Mpc.

each loose group (independently of its richness) in spheres of increasing radius. In a sphere of radius of $2 h^{-1} \mathrm{Mpc} 75$ loose groups have altogether 86 neighbours. This number is comparable with the number of loose groups around each rich cluster sample. We denote this sample as LG.2. In a sphere of radius of $3 h^{-1}$ Mpc already 350 groups have altogether 388 neighbours. We denote this sample as LG.3. Due to the large number of neighbours already in a $3 h^{-1} \mathrm{Mpc}$ sphere we did not use still larger search radii. This actually means that the local number density in the case of these test samples is even higher than in the case of loose groups around rich clusters. 
Table 5. Median and upper quartile (in parentheses) values of LCLG properties.

\begin{tabular}{|c|c|c|c|c|c|c|c|}
\hline Sample & $N_{\text {group }}$ & $N_{\text {obs }}$ & $N_{\mathrm{ACO}}$ & $\begin{array}{r}R_{\mathrm{h}} \\
h^{-1} \mathrm{Mpc} \\
(5)\end{array}$ & $\begin{array}{r}\sigma_{\text {los }} \\
\mathrm{km} \mathrm{s}^{-1} \\
(6)\end{array}$ & $\begin{array}{r}\log M_{\mathrm{vir}} \\
h^{-1} M_{\odot} \\
\text { (7) }\end{array}$ & $\begin{array}{r}\log L_{\mathrm{tot}} \\
h^{-2} L_{\odot} \\
(8)\end{array}$ \\
\hline LCLG.LG.150 & 95 & $4.5(7.0)$ & $19.5(28.0)$ & $0.65(0.89)$ & $235(295)$ & $13.65(14.00)$ & $11.35(11.65)$ \\
\hline LCLG.LG.200 & 65 & $4.5(7.0)$ & $23.5(32.5)$ & $0.74(0.99)$ & $235(280)$ & $13.65(14.00)$ & 11.35 (11.65) \\
\hline LCLG.LG.250 & 57 & $4.5(7.0)$ & $23.5(32.5)$ & $0.62(1.05)$ & $225(290)$ & $13.70(14.05)$ & $11.40(11.65)$ \\
\hline LCLG.cmp.LG.150 & 1223 & $4.0(5.5)$ & $16.0(25.0)$ & $0.46(0.75)$ & $175(265)$ & $13.25(13.70)$ & $11.15(11.35)$ \\
\hline LCLG.cmp.LG.200 & 930 & $4.5(5.5)$ & $16.5(25.5)$ & $0.47(0.80)$ & $185(265)$ & $13.25(13.75)$ & $11.25(11.35)$ \\
\hline LCLG.cmp.LG. 250 & 639 & $4.5(6.0)$ & $17.5(26.0)$ & $0.50(0.87)$ & $185(275)$ & $13.35(13.80)$ & $11.25(11.45)$ \\
\hline
\end{tabular}

The columns are as in Table 3. The lower distance limit is shown in the name of the sample.

Table 6. Median values of the properties of loose groups from samples LG.2, LG.3 and LG.23.

\begin{tabular}{lrrrr}
\hline \hline Sample & LG.2 & LG.3 & LG.23 & LCLG.cmp \\
\hline$N_{\text {group }}$ & 86 & 388 & 302 & 1495 \\
$N_{\text {obs }}$ & 4.0 & 4.0 & 4.0 & 4 \\
$N_{\text {ACO }}$ & 11.0 & 13.0 & 14.0 & 16 \\
$R_{\mathrm{h}}\left(h^{-1} \mathrm{Mpc}\right)$ & 0.37 & 0.44 & 0.46 & 0.58 \\
$\sigma_{\text {los }}\left(\mathrm{km} \mathrm{s}^{-1}\right)$ & 145 & 170 & 185 & 164 \\
$\log M_{\text {vir }}\left(h^{-1} M_{\odot}\right)$ & 13.00 & 13.20 & 13.30 & 13.20 \\
$\log L_{\text {tot }}\left(h^{-2} L_{\odot}\right)$ & 11.05 & 11.10 & 11.00 & 11.10 \\
\hline
\end{tabular}

Moreover, in our samples of loose groups in the neighbourhood of rich clusters, there are no neighbours with centres closer than $2 h^{-1} \mathrm{Mpc}$ to the centre of a rich cluster. Thus, in order to generate a test sample that most closely resembles the samples of loose groups around rich clusters, we used a sample of neighbours from a shell with inner radius $2 h^{-1} \mathrm{Mpc}$ and outer radius $3 h^{-1} \mathrm{Mpc}$. This sample is denoted as LG.23, and there are 302 loose groups in this sample. We note that the distance distribution of groups from these samples is close to that of loose groups in the neighbourhood of rich clusters. There are only 7 loose groups with distances less than $140 h^{-1} \mathrm{Mpc}$ among them.

Then we derived the cumulative distributions of the properties of loose groups from the samples LG.2, LG.3 and LG.23. In Table 6 we give the median values of the properties of groups from these regions of high local number density. The corresponding values for the total sample of the LCLGs are given for comparison (sample LCLG.cmp). Table 6 shows that loose groups from the highest density regions, if the local density is determined by the number density of loose groups themselves independently of their richness, have smaller or comparable parameters when we compare them with parameters of loose groups on average. For the present study the main conclusion from this test is: the properties of loose groups in dense aggregates of loose groups of all richnesses, including the poorest loose groups, are comparable to those for the LCLGs on average, and are not enhanced as are the properties of loose groups in the environment of rich clusters.
In addition, properties of loose groups may be affected by a selection effect related to possible spurious members which may lead to an artificial increase of the richnesses, the velocity dispersions and so on. The tests above do not show the presence of this effect for loose groups from the highest density regions. We also studied the properties of loose groups that are located in the lowest density regions. Loose groups from the lowest density regions can be found using the local number density around loose groups, in analogy with the previous test. Our calculations show that some loose groups from the lowest density regions really have very high velocity dispersions, masses and so on - a hint that the properties of these groups are affected by spurious non-member galaxies. Thus, this selection effect may change the properties of loose groups from the lowest density environments, but not from the high density environments.

Therefore the analysis of various selection effects do not show any indications that selections could cause the artificial enhancement of the properties of loose groups in high density regions in the neighbourhood of rich clusters.

However, selection effects present in the LCRS sample are complicated. It is possible that some of them have not been fully taken into account. Furthermore, this sample of loose groups is probably not complete. Therefore, as a next step, we plan to study the properties of galaxy groups in high density regions using the Sloan Digital Sky Survey (York et al. 2000).

\subsection{Comparison with other studies}

Several recent studies of the correlation function of nearby groups of galaxies indicate that the properties of groups of galaxies in high density regions are different from the properties of groups on average. For example, Giuricin et al. (2001), Girardi et al. (2000), and Merchan et al. (2000) all concluded that groups with larger velocity dispersions and masses are more strongly clustered than groups with lower velocity dispersions and masses. Stronger clustering is an indication that these groups could be located in the high density regions of superclusters (Einasto et al. 1997; Tago et al. 2002).

Other studies have also shown that the properties of groups of galaxies in superclusters may differ from the properties of groups in low density environments. For example, groups in 
superclusters may be X-ray sources (Rines et al. 2001; Bardelli et al. 2000; Kull \& Böhringer 1999).

Using an earlier version of the LCRS loose group catalogue, Hashimoto et al. (1998) have shown that galaxies in massive loose groups show a smaller star formation rate than do galaxies in less massive loose groups. Recently Martinez et al. (2002) and Dominguez et al. (2002) have also shown this effect using a catalogue of loose groups extracted from the $2 \mathrm{dF}$ galaxy redshift survey. They suggest that galaxy populations in more massive groups are more evolved than average populations due to their evolutional history: these galaxies are merger remnants as expected in hierarchical models for galaxy formation. In the future it would be interesting to check the possible connection between their findings and our results.

The studies of the properties of rich clusters of galaxies have also shown evidence that the properties of clusters depend on the large scale environment. Loken et al. (1999) have shown that massive cooling flow clusters are located in high density regions. Additionally, X-ray clusters and clusters with significant substructures (both optical and X-ray clusters) are located preferentially in high density environments (Einasto et al. 2001; Plionis \& Basilakos 2002; Schuecker et al. 2001).

Recently the phenomenon of environmental enhancement has been investigated theoretically using numerical experiments. Faltenbacher et al. (2002) and Gottlöber et al. (2002) have analysed large simulations of the hierarchical formation of galaxies, groups and clusters. They studied the average mass of galaxy and group size dark matter halos in the environment of other galaxies or groups, thereby establishing a clear environmental effect extending up to $10 \mathrm{~h}^{-1} \mathrm{Mpc}$. These authors employed the so-called mark correlation functions (defined in the cited papers) that generalize the cumulative mass functions employed in the present study. The enhanced masses of neighboring halos are connected with early merging processes giving rise to these halos. Therefore, the environmental enhancement of the halo masses is a direct evidence for the process of the hierarchical formation of the galaxy and cluster halos in a network of filaments that connects high density knots of the cosmic mass density.

In the present paper we have analysed deep samples of groups of galaxies and showed that the properties of loose groups in high density environment of rich clusters of galaxies differ from those of groups on average - observational evidence of an environmental enhancement of group properties in the gravitational field of neighbouring rich clusters.

Acknowledgements. We thank Erik Tago and Heinz Andernach for providing us with the compilation of the data about Abell clusters, and Günther Hasinger for providing us with the RBS catalogue. We thank Enn Saar and Sahar Allam for stimulating discussions. The present study was supported by Estonian Science Foundation grant 4695 and by Estonian Research and Development Council grant TO 0060058S98. P.H. was supported by the Finnish Academy of Sciences (grant 46733). J.E. was supported by the German Science Foundation (grant 436 EST 17/2/01). D.L.T. was supported by the US Department of Energy under contract No. DE-AC02-76CH03000. We thank the anonymous referee for useful comments.

\section{Appendix A}

Table A.1. The list of LCLG systems around Abell clusters.

\begin{tabular}{|c|c|c|c|c|c|}
\hline $\begin{array}{r}N_{\text {Abell }} \\
\text { (1) }\end{array}$ & $\begin{array}{r}\alpha \\
(2)\end{array}$ & $\begin{array}{r}\delta \\
(3)\end{array}$ & $\begin{array}{r}D \\
(4)\end{array}$ & $\begin{array}{r}N_{\text {LCLG }} \\
\text { (5) }\end{array}$ & $\begin{array}{r}\text { Near } \\
(6)\end{array}$ \\
\hline Slice & $\delta$ & $-3^{\circ}$ & & & \\
\hline 1098 & 1045 & -340 & 297.1 & 50,51 & \\
\hline 1200 & 1109 & -253 & 294.6 & 71 & \\
\hline 1248 & 1121 & -356 & 319.7 & 85 & \\
\hline 1308 & 1130 & -342 & 178.2 & $88,92,94,101,106$ & \\
\hline 1386 & 1148 & -140 & 283.7 & 125 & $\mathrm{~N}$ \\
\hline 1399 & 1148 & -249 & 253.1 & $122,127,128$ & \\
\hline 1404 & 1149 & -232 & 251.6 & 128,134 & $\mathrm{~N}$ \\
\hline 1651 & 1256 & -355 & 236.7 & 172 & \\
\hline 1658 & 1258 & -310 & 237.3 & $172,174,176$ & \\
\hline 1663 & 1300 & -215 & 231.9 & 174,176 & \\
\hline 1729 & 1321 & -306 & 316.9 & 185 & \\
\hline 2045 & 1511 & -234 & 284.0 & $277,278,281$ & \\
\hline Slice & $=$ & $-6^{\circ}$ & & & \\
\hline 930 & 1004 & -523 & 235.9 & 4 & \\
\hline 978 & 1018 & -616 & 154.8 & $10,13,14$ & \\
\hline 1009 & 1022 & -532 & 275.9 & 16 & \\
\hline 1214 & 1114 & -520 & 281.4 & 42 & \\
\hline 1448 & 1200 & -633 & 347.9 & 70,72 & \\
\hline 1482 & 1208 & -518 & 221.5 & 75,78 & \\
\hline Slice & $\delta=$ & $-12^{\circ}$ & & & \\
\hline 970 & 1015 & -1027 & 168.6 & 7,8 & $\mathrm{~N}$ \\
\hline 1309 & 1130 & -1134 & 281.6 & 80,85 & \\
\hline 1348 & 1138 & -1205 & 323.5 & 88,94 & \\
\hline 1606 & 1242 & -1143 & 264.9 & 142,144 & \\
\hline 1754 & 1329 & -1124 & 224.2 & 185 & \\
\hline 1796 & 1347 & -1140 & 245.2 & 200,203 & \\
\hline 1837 & 1359 & -1055 & 198.1 & $212,213,218$ & \\
\hline 2031 & 1509 & -1100 & -308.2 & 270,271 & $\mathrm{~N}$ \\
\hline Slice & $\delta=$ & $-39^{\circ}$ & & & \\
\hline 2717 & 0001 & -3614 & 141.7 & 255 & $\mathrm{~N}$ \\
\hline 2767 & 0020 & -3824 & 328.3 & 8,9 & \\
\hline 2772 & 0022 & -3816 & 327.3 & 9 & \\
\hline 2799 & 0035 & -3924 & 180.9 & 18 & \\
\hline 2856 & 0059 & -3856 & 336.1 & 28,29 & \\
\hline 2860 & 0101 & -4003 & 214.8 & 33 & \\
\hline 2911 & 0123 & -3814 & 218.8 & 43,48 & \\
\hline 2960 & 0157 & -3816 & 286.8 & 57 & \\
\hline 3135 & 0332 & -3910 & 178.2 & $106,111,122,123$ & \\
\hline 3142 & 0334 & -3958 & 186.7 & 123 & \\
\hline 3145 & 0336 & -3811 & 199.2 & 120 & \\
\hline 3984 & 2312 & -3804 & 261.5 & $225,226,227$ & \\
\hline 4008 & 2327 & -3936 & 158.1 & 236 & \\
\hline 4021 & 2335 & -3823 & 296.1 & 239,241 & \\
\hline 4029 & 2341 & -3833 & 284.2 & 244 & \\
\hline 4068 & 2357 & -3945 & 284.5 & 249 & \\
\hline Slice & $\delta=$ & $-42^{\circ}$ & & & \\
\hline 2718 & 0001 & -4213 & 343.7 & 6 & \\
\hline 3122 & 0320 & -4131 & 182.6 & 95 & \\
\hline 3756 & 2112 & -4249 & 215.9 & 148,150 & \\
\hline 3772 & 2126 & -4258 & 198.4 & 151 & \\
\hline 3775 & 2128 & -4332 & 291.5 & 156 & $\mathrm{~N}$ \\
\hline 3920 & 2246 & -4111 & 345.9 & 212 & \\
\hline 3998 & 2318 & -4210 & 252.1 & 236,237 & \\
\hline
\end{tabular}


Table A.1. continued.

\begin{tabular}{rrrrrr}
\hline \hline $\begin{array}{r}N_{\text {Abell }} \\
(1)\end{array}$ & $\begin{array}{r}\alpha \\
(2)\end{array}$ & $\begin{array}{r}\delta \\
(3)\end{array}$ & $\begin{array}{r}D \\
(4)\end{array}$ & $\begin{array}{r}N_{\text {LCLG }} \\
(5)\end{array}$ & $\begin{array}{r}\text { Near } \\
(6)\end{array}$ \\
\hline Slice & $\delta$ & $=$ & $-45^{\circ}$ & & \\
\hline 3102 & 0312 & -4449 & 319.7 & 110 & \\
3104 & 0312 & -4536 & 206.7 & 113 & \\
3111 & 0316 & -4555 & 218.3 & 114 & \\
3112 & 0316 & -4425 & 211.6 & 114 & \\
3133 & 0331 & -4607 & 198.4 & 120 & \\
3749 & 2106 & -4601 & 189.1 & 159 & \\
3754 & 2112 & -4541 & 273.8 & 157 & \\
3757 & 2115 & -4527 & 267.6 & 167 & \\
3809 & 2143 & -4408 & 177.7 & 184 & \\
3908 & 2242 & -4518 & 252.1 & $217,219,223$ & \\
3910 & 2243 & -4615 & 252.9 & 219,221 & $\mathrm{~N}$ \\
3963 & 2301 & -4435 & 200.0 & 236,240 & \\
3970 & 2303 & -4529 & 345.4 & 239 & \\
\hline
\end{tabular}

Endnotes: the columns in Tables 5.3,1 and 2 are as follows: Column 1: Number of the cluster from Abell catalogue (Table 5.3), from APM catalogue (Table 1) and from the RBS catalogue (Table 2; $R x$ - number from the catalogue by de Grandi et al. 1999).

Column 2 and 3: the right ascension (in HH MM format) and declination (in DD MM format, equinox 1950) of rich cluster.

Column 4: distance of rich cluster, in units of $h^{-1} \mathrm{Mpc}$.

Column 5: LCLG catalogue numbers of loose groups around clusters. Column 6: Tables 5.3 and 1: index $N$ (for Near) for those clusters that have close groups among LCLGs, but which themselves are located outside of LCRS slice boundaries. Table 2: the Abell number of the $\mathrm{X}$-ray cluster (nA - non-Abell cluster).

\section{References}

Abell, G. 1958, ApJS, 3, 211

Abell, G., Corwin, H., \& Olowin, R. 1989, ApJS, 70, 1

Andernach, H., \& Tago, E. 1998, in Large Scale Structure: Tracks and Traces, ed. V. Müller, S. Gottlöber, J. P. Mücket, \& J. Wambsganss (Singapore: World Scientific), 147

Bardelli, S., Zucca, E., Zamorani, G., Moscardini, L., \& Scaramella, R. 2000, MNRAS, 312, 540

Borgani, S., \& Guzzo, L. 2001, Nature, 409, 39

Carlberg, R. G., Yee, H. K. C., \& Ellingson, E. 1997, ApJ, 478, 462

Carlberg, R. G., Yee, H. K. C., Morris, S. L., et al. 2001, ApJ, 552, 427

Colless, M., Dalton, G., Maddox, S., et al. 2001, MNRAS, 328, 1039

Dalton, G. B., Maddox, S. J., Sutherland, W. J., \& Efstathiou, G. 1997, MNRAS, 289, 263 (D97)

de Grandi, S., Böhringer, H., Guzzo, L., et al. 1999, ApJ, 514, 148

Dominguez, M., Zandivarez, A., Martinez, H. J., et al. 2002, MNRAS, 335,825
Doroshkevich, A. G., Tucker, D. L., Fong, R., Turchaninov, V., \& Lin, H. 2001, MNRAS, 322, 369

Einasto, J., Einasto, M., Frisch, P., et al. 1997, MNRAS, 289, 801

Einasto, M. 1991, MNRAS, 252, 261

Einasto, M., Einasto, J., Tago, E., Müller, V., \& Andernach, H. 2001, AJ, 122, 2222

Einasto, M., Tago, E., Einasto, J., et al. 2002, AJ, 123, 51

Faltenbacher, A., Gottlöber, S., Kerscher, M., \& Müller, V. 2002, A\&A, 295, 1

Girardi, M., Giuricin, G., Mardirossian, F., Mezzetti, M., \& Boschin, W. 1998, ApJ, 505, 74

Girardi, M., Boschin, W., \& da Costa, L. N. 2000, A\&A, 353, 57

Giuricin, G., Samurovic, S., Girardi, M., Mezzetti, M., \& Marinoni, C. 2001, ApJ, 554, 857

Gottlöber, S., Kerscher, M., Klypin, A., Kravtsov, A., \& Müller, V. 2002, A\&A, 387, 778

Guzzo, L., Böhringer, H., Schuecker, P., et al. 1999, The Messenger, 95,27

Hamilton, A. J. S. 1988, ApJ, 331, L59

Hashimoto, Y., Oemler, A., Lin, H., \& Tucker, D. 1998, ApJ, 499, 589

Heinämäki, P., Einasto, J., Einasto, M., et al. 2003, A\&A, 397, 63

Kull, A., \& Böhringer, H. 1999, A\&A, 341, 23

Lindner, U., Einasto, J., Einasto, M., et al. 1995, A\&A, 301 , 329

Loken, C., Melott, A. L., \& Miller, C. J. 1999, ApJ, 520, L5

Maddox, S. J., Efstathiou, G., \& Sutherland, W. J. 1996, MNRAS, 283,1227

Martinez, H. J., Zandivarez, A., Dominguez, M., Merchan, M. E., \& Lambas, D. G. 2002, MNRAS, 333, 31

Mattig, W. 1958, Astron. Nachr., 284, 109

Mazure, A., Katgert, P., den Hartog, R., et al. 1996, A\&A, 310, 31

Merchan, M., Maia, M. A. G., \& Lambas, D. G. 2000, ApJ, 545, 26

Merchan, M., \& Zandivarez, A. 2002, MNRAS, 335, 216

Norberg, P., Baugh, C. M., Hawkins, E., et al. 2001, MNRAS, 328, 64

Peacock, J. A., \& West, M. J. 1992, MNRAS, 259, 494

Plionis, M., \& Basilakos, S. 2002, MNRAS, 329, L47

Ramella, M., Zamorani, G., Zucca, E., et al. 1999, A\&A, 342, 1

Rines, K., Mahdavi, A., Geller, M. J., et al. 2001, ApJ, 555, 558

Schuecker, P., Boehringer, H., Reiprich, T. H., \& Feretti, L. 2001, A\&A, 378, 408

Schwope, A. D., Hasinger, G., Lehmann, I., et al. 2000, Astron. Nachr., 321, 1 (RBS)

Shectman, S., Landy, S., Oemler, A., et al. 1996, ApJ, 470, 122

Tago, E., Saar, E., Einasto, J., et al. 2002, AJ, 123, 37

Trümper, J. 1993, Science, 260, 1769

Tucker, D. L., Oemler, A. Jr., Hashimoto, Y., et al. 2000, ApJS, 130, 237 (TUC)

Vettolani, G., Zucca, E., Zamorani, G., et al. 1997, A\&A, 325, 954

Voges, W., Aschenbach, B., Boller, T., et al. 1999, A\&A, 349, 389

York, D. G., Adelman, J., Anderson, J. E., et al. 2000, AJ, 120, 1579

Zehavi, I., Blanton, M. R., Frieman, J. A., et al. 2002, ApJ, 571, 172 